Europhysics Letters

PREPRINT

\title{
Crossover from stationary to aging regime in glassy dy- namics
}

\author{
A. Andreanov ${ }^{1}$ and A. LefÈvre ${ }^{1}$ \\ 1 Service de Physique Théorique, Orme des Merisiers - CEA Saclay, 91191 Gif-sur- \\ Yvette Cedex, France.
}

\begin{abstract}
We study the non-equilibrium dynamics of the spherical p-spin models in the scaling regime near the plateau and derive the corresponding scaling functions for the correlators. Our main result is that the matching between different time regimes fixes the aging function in the aging regime to $h(t)=\exp \left(t^{1-\mu}\right)$. The exponent $\mu$ is related to the one giving the length of the plateau. Interestingly $1-\mu$ is quickly very small when one goes away from the dynamic transition temperature in the glassy phase. This gives new light on the interpretation of experiments and simulations where simple aging was found to be a reasonable but not perfect approximation, which could be attributed to the existence of a small but non-zero stretching exponent.
\end{abstract}

Introduction. - Glassy phases are widely observed in Nature and in materials [1]. The most stricking features are very slow relaxations and history dependent phenomena, such as rejuvenation or memory $[2,3]$. Such phenomena reflect that the system is aging: the older the system, the slower the relaxation. The arrest of the dynamics is generally attributed to large scale freezing, and the approach to the glass transition has recently been shown to be associated with strong dynamic heterogeneity [4]. Some regions of space may relax very fast, while others may relax very slowly. In order to relax almost frozen regions, many particles have to move cooperatively, and the dynamical arrest arises when the time for relaxing large regions - the relaxation time - exceeds the available experimental time. The understanding of the freezing phenomenon and of the low temperature - or glassy - phase has been boosted by the introduction and the resolution of models such as the spherical p-spin model, or the mode-coupling theory [5]. In these models, the dynamics stops at some critical temperature $T_{c}$, the idealised glass transition temperature. The dynamics of the spherical p-spin model has been solved in the limit of very large waiting times [6], which lead to the following phenomenology in the glassy phase. In this phase a sharp separation of timescales occurs, with two distinct time sectors [7], which in turn correspond to fast and slow degrees of freedom. The former correspond to local (in time) equilibration of local (in space) degrees of freedom and is often pictured by a rattling inside "cages", which respond to a small external perturbating field through Fluctuation Dissipation Theorem (FDT). The latter rule the slow structural relaxation, following a slow descent in the energy landscape [8], and their response can be described using a modified FDT, in which the temperature $T$ is replaced by an effective temperature $T^{*} \geq T$. Still, old samples exhibit stationarity on finite time differences. Another

(C) EDP Sciences 
striking consequence of the analytic solution of the p-spin model in the limit of an infinite waiting time is that for very old samples the correlation function $C\left(t, t^{\prime}\right)$ (we will always choose $t>t^{\prime}$, with $t$ written on the left) takes the form $\mathcal{C}\left(\frac{h\left(t^{\prime}\right)}{h(t)}\right)$ when $t \rightarrow \infty$ with $t \sim t^{\prime}$. This form has been used to fit data in experiments and simulations of aging systems. In many cases, $h(t)$ is fairly well approximated by a power law ("simple aging") $[9,10]$; however better fits have often been obtained by using $h(t)=\exp \left(t^{1-\mu} /(1-\mu)\right)$, with $\mu<1$ but $\mu$ close to 1 ("sub aging") $[9,11,12]$. The interpretation was that the times used for measurements were two small and that longer waiting times would provide better agreement with simple aging. However, the study of the p-spin (or related) models has not helped resolving this issue, as in this case $h(t)$ is left unknown by the dynamic equations in the aging regime. In other words, in the aging regime, the dynamics is invariant under strictly increasing time reparametrizations $[6,13]$.

In this Letter, we revisit the analytic solution of [6], by unveiling a new time scale, which as we shall show is crucial in order to understand the crossover from the stationary to the aging regime, and which we shall call the "scaling regime". It corresponds to times of the order $t_{w}^{\beta}$, where $t_{w}$ is the waiting time and $\beta$ a new exponent, and was studied in detail in the spherical Sherrington-Kirkpatrick (SK) model in [17]. Our main results are the calculation of the aging function $h(t)$ and the description of the large but finite waiting time behaviour of the dynamic correlators.

The model. - We focus on the celebrated spherical p-spin model is defined by the Hamiltonian $H_{p}=\sum_{1 \leq i_{1} \cdots i_{p} \leq N} J_{i_{1} \cdots i_{p}} \phi_{i_{1}} \cdots \phi_{i_{p}}$, where $N$ is the number of soft spins, which verify the spherical constraint $\sum_{i} \phi_{i}^{2}=N$ and the $J_{i_{1} \cdots i_{p}}$ 's are Gaussian random coupling with vanishing average and variance $N^{p-1} / p$ ! . Except when specified, we shall consider only $p>2$, where a discontinuous ideal glass transition occurs [14]. The dynamic equations for these models in the large $N$ limit involve the correlator $C\left(t, t^{\prime}\right)=\left\langle\phi_{i}(t) \phi_{i}\left(t^{\prime}\right)\right\rangle$ and the response $R\left(t, t^{\prime}\right)=\left\langle\frac{\delta \phi_{i}(t)}{\delta \eta_{i}\left(t^{\prime}\right)}\right\rangle$ to an external field $\eta_{i}$. It is convenient to use the integrated response $F\left(t, t^{\prime}\right)=\int_{t^{\prime}}^{t} d s R(t, s)$, which leads to the following equations [7]:

$$
\begin{aligned}
& \left(\partial_{t}+z(t)\right) F\left(t, t^{\prime}\right)=1+\int_{t^{\prime}}^{t} d s \Sigma(t, s) F\left(s, t^{\prime}\right), \\
& \left(\partial_{t}+z(t)\right) C\left(t, t^{\prime}\right)=\int_{0}^{t} d s \Sigma(t, s) C\left(t^{\prime}, s\right)+\int_{0}^{t^{\prime}} d s D(t, s) \partial_{s} F\left(t^{\prime}, s\right),
\end{aligned}
$$

where $\Sigma(t, s)=-\frac{p(p-1)}{2} C(t, s)^{p-2} \partial_{s} F(t, s), D(t, s)=\frac{p}{2} C(t, s)^{p-1}$ and the Lagrange multiplier $z(t)=T+\frac{p^{2}}{2} \int_{0}^{t} d s C(t, s)^{p-1} \partial_{s} F(t, s)$ enforces the constraint $\sum_{i} \phi_{i}(t)^{2}=N$. There is no characteristic timescale in Eqs. (112), as the time is expressed in units of the microscopic timescale $t_{0}$.

The different time regimes. - In the limit of large waiting time $t^{\prime} \rightarrow \infty$, the dynamics can be partly solved assuming the sharp separation between the stationary and aging regimes [7]. In this limit, when $t-t^{\prime} \sim 1$, the solution of (11) and (2) is stationary: $C\left(t, t^{\prime}\right)=C_{S T}\left(t-t^{\prime}\right)$ and $R\left(t, t^{\prime}\right)=R_{S T}\left(t-t^{\prime}\right)$, where $C_{S T}$ and $R_{S T}$ are related by FDT: $T R_{S T}(\tau)=-d C_{S T}(\tau) / d \tau$ $(\tau>0)$. The stationary part of the correlation behaves at large $\tau$ as $C_{S T}(\tau)=q_{E A}+$ $c_{S T}^{(1)} \tau^{-a}+c_{S T}^{(2)} \tau^{-2 a}+\cdots$, where $a$ verifies $\frac{\Gamma(1-a)^{2}}{\Gamma(1-2 a)}=T /\left(2 T^{*}\right)$. On the other hand, when $t-t^{\prime} \sim t^{\prime}$, the solution has the form [6] $C\left(t, t^{\prime}\right)=\mathcal{C}\left(\frac{h\left(t^{\prime}\right)}{h(t)}\right), F\left(t, t^{\prime}\right)=\mathcal{F}\left(\frac{h\left(t^{\prime}\right)}{h(t)}\right)$, which satisfy $\mathcal{F}(\lambda)=\frac{1-\mathcal{C}(\lambda)}{T^{*}}$. The effective temperature $T^{*}$ verifies $T / T^{*}=\frac{(p-2)\left(1-q_{E A}\right)}{q_{E A}}$, while 
the Edwards-Anderson parameter satisfies $\frac{p(p-1)}{2} q_{E A}^{p-2}\left(1-q_{E A}\right)^{2}=T^{2}$. Near $\lambda=1: \mathcal{C}(\lambda)=$ $q_{E A}-c_{A G}^{(1)}(1-\lambda)^{b}-c_{A G}^{(2)}(1-\lambda)^{2 b}+\cdots$, where $b=1$ for the spherical $\mathrm{p}(>2)$-spin model (we keep $b$ when possible, as some of the results apply to a wider class of models) [15].

Now we add an extra timescale to the picture. We assume the existence of an exponent $\beta$ which governs the size of the plateau with $t^{\prime}$, or equivalently $t$. We describe time differences of order $t^{\beta}$, interpolating between the stationary and aging regimes, thus $\beta<1$. The existence of this scaling regime is confirmed by numerics [16] and by the analytic solution in the case $p=2[17]$. The thickness of the plateau is described by another exponent $\alpha$ such that $C\left(t, t^{\prime}\right)=$ $q_{E A}+t^{-\alpha} g_{1}\left(\frac{t-t^{\prime}}{t^{\beta}}\right)+t^{-2 \alpha} g_{2}\left(\frac{t-t^{\prime}}{t^{\beta}}\right)+\cdots$ and $F\left(t, t^{\prime}\right)=\frac{1-q_{E A}}{T}+t^{-\alpha} w_{1}\left(\frac{t-t^{\prime}}{t^{\beta}}\right)+t^{-2 \alpha} w_{2}\left(\frac{t-t^{\prime}}{t^{\beta}}\right)+\cdots$. This implies $R\left(t, t^{\prime}\right)=t^{-\gamma} r_{1}\left(\frac{t-t^{\prime}}{t^{\beta}}\right)+t^{-\gamma-\alpha} r_{2}\left(\frac{t-t^{\prime}}{t^{\beta}}\right)+\cdots$, with $\gamma=\alpha+\beta$, and $r_{i}(x)=$ $\partial w_{i}(x) / \partial x$. In addition, one also introduces $\tilde{T}(x)=-g_{1}^{\prime}(x) / w_{1}^{\prime}(x)$, which goes from $T$ at small $x$ to $T^{*}$ at large $x$; the finiteness of $\tilde{T}(x)$ explains the occurrence of the same $\alpha$ for $C$ and $F$.

Matching the different regimes. - The scaling regime interpolates between the stationary regime at small arguments and the aging regime at large arguments. This gives relations between different exponents and scaling laws. First we take $\tau=t-t^{\prime}=\epsilon t^{\beta}$, with $t \rightarrow \infty$ and $\epsilon \ll 1$ (in this order). Matching with the stationary regime gives $t^{-\alpha} g_{i}(\epsilon) \sim_{\epsilon \rightarrow 0} c_{S T}^{(i)} \epsilon^{-i a} t^{i \beta a}$, and thus $g_{i}(\epsilon) \sim c_{S T}^{(i)} \epsilon^{-i a}, \alpha=\beta a$. It also gives $w_{i}(\epsilon) \sim-c_{S T}^{(i)} / T \epsilon^{-i a}$. This is similar to the analysis around the plateau in the high temperature phase [5]. Second, we take $\tau=x t^{\beta}$ with $x \gg 1$ and match with the aging regime. On one hand $C\left(t, t^{\prime}\right)=\mathcal{C}\left(\frac{h\left(t^{\prime}\right)}{h(t)}\right)=q_{E A}-$ $c_{A G}^{(1)}(\tau \phi(t))^{b}-\cdots$, with $\phi(t)=\frac{d \ln h(t)}{d t}$. On the other hand $C\left(t, t^{\prime}\right)=q_{E A}+t^{-\alpha} g_{1}(x)+\cdots$. Comparing the dependences in $\tau$ and $t$ gives $g_{i}(x) \sim-c_{A G}^{(i)}\left(A_{i} x\right)^{b i}, w_{i}(x) \sim c_{A G}^{(i)}\left(A_{i} x\right)^{b i} / T^{*}$ and $\phi(t) \sim t^{-\mu} / A_{i}$, with $\mu=\beta+\alpha / b$. On can conclude that $h(t) \sim \exp \left(\frac{t^{1-\mu}}{A_{1}(1-\mu)}\right)$, provided $\phi(t)-t^{-\mu} \ll 1 / t$. The corrections to $t^{-\mu}$ are given by the corrections to the leading order of the $w_{i}$ 's $(i \geq 2)[18]$ and can be neglected when $\mu+\alpha>1$. There is numerical evidence (see below) that this is the case for p-spin models, and thus $\phi(t)=t^{-\mu} / A_{1}+o\left(t^{-1}\right)$. This gives $h(t) \sim \exp \left(\frac{t^{1-\mu}}{A_{1}(1-\mu)}\right)$, and thus $0 \leq \mu \leq 1$, as $h(t)$ must map infinity onto infinity. We set $A_{1}$ to 1 , as changing $A_{1}$ to $A_{1}^{\prime}$ is equivalent to changing $\mathcal{C}(\lambda)$ to $\mathcal{C}\left(\lambda^{A_{1} / A_{1}^{\prime}}\right)$. This freedom to choose $A_{1}$ reflects that the miscroscopic timescale is forgotten in the aging regime. It provides further support for the absence of non-vanishing (except logarithmic) corrections to $\ln h(t) \sim t^{1-\mu} /(1-\mu)$. Indeed, $\ln h(t)=t^{1-\mu} /(1-\mu)+A_{2} t^{\delta}+\cdots$, with $\delta>0$ would fix the microscopic timescale $t_{0}$ through $A_{2} \propto t_{0}^{-\delta}$. This argument can even be used to give directly the form of $h(t)$. Indeed, assuming $\mathcal{C}(\lambda)=\lambda^{\nu\left(t_{0}\right)}, h(t)=\exp f\left(t / t_{0}\right)$ and $\partial_{t_{0}} C\left(t, t^{\prime}\right)=0$ in the aging regime gives $f(u)=A\left(t_{0}\right)+B\left(t_{0}\right) u^{c}$ [18]. Thus $h$ is either a stretched exponential or a power law, while other empirical forms [12] are ruled out. Remark that in order to obtain $h(t)$ by matching the different regimes, we have integrated the large time behaviour, so this in principle gives $h(t)$ only approximately. However the meaning of the aging function is only asymptotic, and valid for large $t$. Note that the relations we have derived between exponents are very general, provided the expansions we used for the late stationary and early aging regimes are valid.

Equations for the scaling regime. - Now we sketch the - lengthy - derivation of the equations for the scaling functions at the plateau, which will be detailed elsewhere [18]. The following procedure gives equations for $g_{1}$ and $w_{1}$, but the exponents $\alpha$ and $\beta$ go away, and $\beta$ remains unknown. We set $t-t^{\prime}=x t^{\beta}$ in (11) and (2) and separate the integrals in different 
time sectors. Then, the identification of different orders gives the equations. In the mean time, one gets for large times $z(t)=z(\infty)-z_{1} t^{-\alpha}-z_{2} t^{-2 \alpha}-\cdots$, where $z_{1}=0$ and $z_{2}>0$ [18]. The multiplier $z(t)$ being a one-time quantity, its value is rather easy to get in numerics, and thus provides a useful estimate for $\alpha$; in addition, it also gives the behaviour of the energy density $\mathcal{E}(t)=(T-z(t)) / p$. Now we write $\int_{0}^{t^{\prime}} d s=\int_{0}^{\eta t}+\int_{\eta t}^{t^{\prime}-\Lambda t^{\beta}}+\int_{t^{\prime}-\Lambda t^{\beta}}^{t^{\prime}-\epsilon t^{\beta}}+\int_{t^{\prime}-\epsilon t^{\beta}}^{t^{\prime}}$, which separates $t-s$ in several distinct regimes: very short waiting times, aging, scaling and stationary. Next we take the limit $t \rightarrow \infty$, followed by $\eta \rightarrow 0, \Lambda \rightarrow \infty$ and $\epsilon \rightarrow 0$. We then identify all orders in $t^{-\alpha}$ : taking the above limits leads to divergences which cancel each other giving en passant the values of the coefficients of the leading orders of the $g_{i}$ 's and $w_{i}$ 's $(i \geq 2)$. We also write $\int_{t^{\prime}}^{t}=\int_{t^{\prime}}^{t^{\prime}+\epsilon_{1} t^{\beta}}+\int_{t^{\prime}+\epsilon_{1} t^{\beta}}^{t-\epsilon_{2}}+\int_{t-\epsilon_{2} t^{\beta}}^{t}$ and send $\epsilon_{1}$ and $\epsilon_{2}$ to zero. We now give the results of the identification of different orders. First, the orders $t^{0}$ and $t^{-\alpha}$ of (12) give: $z(\infty)=\frac{p(p-1)}{2} q_{E A}^{p-2}\left(1-q_{E A}\right)+\frac{1-q_{E A}^{p-1}}{T}$, and we get at order $t^{-2 \alpha}$ :

$$
\begin{array}{r}
w(x)^{2}+\int_{x_{0}}^{x} d y w^{\prime}(y) \frac{g(y)}{T^{*}}+\int_{0}^{x} d y w^{\prime}(y)(w(x-y)-w(x))=C_{F}, \\
\int_{x_{0}}^{\infty} d y\left[(p-1) g(y)\left(w^{\prime}(x+y)-w^{\prime}(y)\right)+w^{\prime}(y)(g(x+y)-g(y))\right] \\
+(p-2) \int_{0}^{\infty} d y w^{\prime}(x+y)(g(x+y)-g(y))+\int_{x_{0}}^{x} d y w^{\prime}(y)(g(x-y)+(p-2) g(y)) \\
+\int_{0}^{x_{0}} d y\left[(p-1) g(y) w^{\prime}(x+y)+w^{\prime}(y)(g(x+y)+g(x-y)-2 g(x))\right] \\
+\frac{g(x)^{2}}{2 T^{*}}+2 w\left(x_{0}\right) g(x)-\frac{(p-1) \nu}{T^{*}} q_{E A} g_{\infty} x+\frac{(p-2) q_{E A}^{2} \nu^{2}}{2 T^{*}} x^{2}=C_{C},
\end{array}
$$

where $x_{0}$ is such that $g\left(x_{0}\right)=0$, and $g_{\infty}, C_{F}$ and $C_{C}$ will be given somewhere else [18] $(\nu$ is given below). In order to obtain consistent equations, we had to make the assumption that $C\left(t, t^{\prime}\right)$ vanishes faster than $t^{-2 \alpha}$ when $t^{\prime}$ is finite and $t \rightarrow \infty$. This assumption is consistent with the stretched exponential behaviour of $h(t)$, and has been checked numerically. We thus ended up with coupled equations for $g_{1}$ and $w_{1}$. It can be easily checked that (3) is compatible with the asymptotic behaviours obtained from the matching with the other regimes; the same check for (4) is harder. In addition, integrating these equations from small to large $x$ in principle provides the value of $c_{A G}^{(1)}$. The p-spin model is a special case where the form of the aging scaling function is known ; indeed one has simply [6]: $\mathcal{C}(\lambda)=q_{E A} \lambda^{\nu}$, which gives $c_{A G}^{(1)}=-\nu$ (recall $A=1$ ). Thus, $\mathcal{C}$ is in principle determined by $g_{1}$ and $w_{1}$, although we have not yet been able to solve (34) analytically or numerically.

The case $p=2$. - For $p=2, C\left(t, t^{\prime}\right)$ and $R\left(t, t^{\prime}\right)$ can be computed exactly [19], and $T^{*}=\infty$. In addition the exponents of the scaling regime are [17] $\beta=4 / 5$ and $\alpha=2 / 5$. Moreover the analysis must be slightly refined [18], as $g_{1}=0$ and correspondly $c_{A G}^{(1)}=0$; it gives $\mu=1$, which corresponds to simple aging, as confirmed by the exact solution. Remark that here one has $z_{2}=0$, while $z_{3}=-3 / 8$.

Numerical check. - As one exponent is still unknown, we have checked the relations between different exponents using a code for numerical integration of the equations provided by the authors of [20]. We start with $p=3$. At $T=0.5$, we obtained $\alpha \approx 0.3$ from the fit of $z(t)$, and $\beta \approx 0.66$ from the scaling of $t_{\beta}\left(t^{\prime}\right)$ which solves $C\left(t^{\prime}+t_{\beta}\left(t^{\prime}\right), t^{\prime}\right)=q_{E A}$. This is in very good agreement with $\alpha=\beta a(a \approx 0.448)$ and gives $\mu \approx 0.96$, which provides a very good collapse of the data in the aging regime, as found empirically in [16]. The same test was 


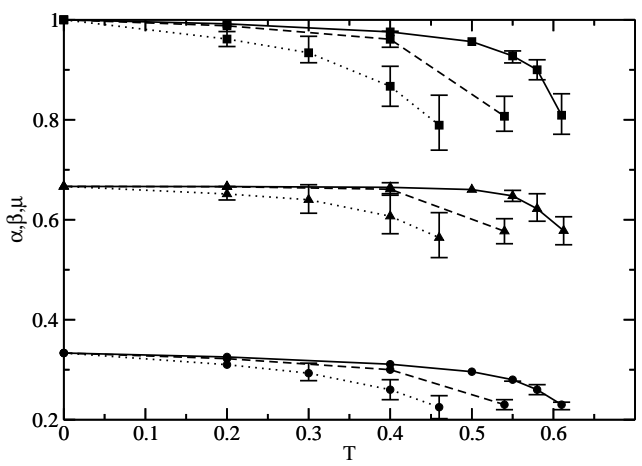

Fig. 1 - Exponents $\alpha$ (circles), $\beta$ (triangles) and $\mu$ (squares) versus temperature for $p=3$ (solid lines), $p=4$ (dashed lines) and $p=10$ (dotted lines).

carried out at several temperatures from $T=0$ to $T=0.61\left(T_{c}=\sqrt{3 / 8} \approx 0.6125\right)$, providing very good agreement with our analytic description. In Fig. 1 the exponents $\alpha, \beta$ and $\mu$ are shown (along with the same for $p=4$ and $p=10$ ). The observed tendency is that they decrease with $p$ and $T$. In addition, $\beta$ and $\mu$ saturate respectively to $2 / 3$ and 1 , their values at $T=0$. The value $\mu(T=0)=1$ corresponds to approximate aging slightly above $T=0$. It is clear from Fig. 1 that $\mu+\alpha>1$ is a reliable working assumption. Finally, we have checked numerically the collapse of the data when using the scaling we introduced for the plateau regime: in Fig 2. $-T \partial F / \partial C$ is represented versus $\left(C\left(t^{\prime}+\tau, t^{\prime}\right)-q_{E A}\right) t^{\prime \alpha}$ parameterised by $\tau$ for several waiting times, for $p=3$ and $T=0.5$. A consequence of our scaling hypothesis is that a master curve is obtained, identical to the curve of $T / \tilde{T}(x)$ versus $g(x)$, which shows the onset of FDT violation during the scaling regime.

Discussion. - In many glassy systems, fits of the aging regime were done using $h(t) \sim t$ (or any power law), giving reasonable agreement. It was however observed that $h(t) \sim$ $\exp \left(t^{1-\mu}\right)$ gives better collapse of the thermo-remanent magnetisation in spin-glass experiments, with $\mu$ slightly below $1[9,11]$. An interpretation of this fact was that the waiting time was not large enough for the aging scaling to be seen well. In the case of the p-spin model, as we have shown, the stretched exponential form is related to the power law dependance of the timescale of the plateau with the waiting time and to the forgetting of the microscopic timescale in the aging regime. In addition, we have found $\mu<1$ from numerical estimates, which appears as an evidence against simple aging. However $\mu$ departs significantly from 1 in the vicinity of the critical point, and the numerical estimation of the exponents may be flawed by critical effects. For instance, the exponents shown on Fig. 1 seem to have a cusp at $T_{c}$, which could be an indication that the scaling regime is strongly influenced by the cusp of $q_{E A}$ at $T_{c}$ [5], as the position of the plateau itself fluctuates a lot at the approach to $T_{c}$. A simple way to discriminate such effects would be to compute the variation of $\beta$ with $T$. Indeed our numerical results suggest that $\beta$ decreases with $T$, while $\beta(T)$ obtained by imposing $\mu=1$ at all temperatures - which gives $\beta(T)=1 /(1+a(T))$ - is an increasing function. It remains that for fixed $t^{\prime}$, our data and those of [16] are clearly compatible with stretched exponentials rather than power laws. The scenario $\mu<1$ is at least relevant for the time regimes numerically accessible. 


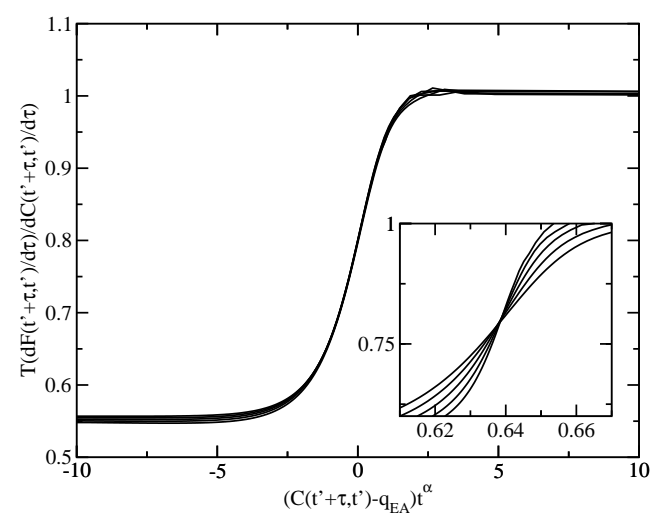

Fig. 2 - Test of the scaling hypothesis for $p=3$ and $T=0.5$. Inset: same as main, but with $C\left(t^{\prime}+\tau, t^{\prime}\right)$ as horizontal coordinate ; the crossing occurs at $q$ and corresponds to $\tau=x_{0} t^{\beta}$, with $g\left(x_{0}\right)=0$. Waiting times are $t^{\prime}=2197, t^{\prime}=4295, t^{\prime}=8590, t^{\prime}=17180$ and $t^{\prime}=34359$.

Conclusion. - In this Letter, we have shown that the matching of the stationary and aging regimes in glassy systems requires the precise description of the intermediate scaling regime, which is defined at large but finite waiting time. We have shown that the natural assumption that in this regime the correlators obey power law scaling leads to a stretched exponential form for the aging function. In addition, the exponent is related to the one giving the duration of the plateau. The assumptions and ansätze which have been made in order to get information about the scaling regime have been checked with very good accuracy using numerical integration of the exact dynamic equations. The consequences of these results are threefold. First, it shows the p-spin model as an archetype system where the aging function is close to a power law, but different, at least in the accessible time regimes. This provides a motivation for revisiting experimental and numerical data fits where the same phenomenon was observed. Second, our calculation provides explicit corrections at large but finite times to the solution given in [6]. This may indicate the route to follow in order to study the dynamics of systems where the ideal transition does not really exist, and where an aging behaviour, with a waiting time dependent effective temperature was found. Here a calculation at large, but not infinite waiting time is clearly necessary. Third, we hope that our treatment may lead to a proper mathematical approach to the solution of the dynamic equations. Indeed, it has recently been proven rigorously [21] that the dynamic equations governing the dynamics in the (thermodynamic) limit $N \rightarrow \infty$ derived in [6]. The important challenge is now to compute the exponent $\beta$, i.e. the length of the plateau. Indeed, it is important to confirm whether or not $\mu<1$ is not a numerical effect due to critical fluctuations. Finally, our analysis remains to be adapted to models with continuous replica symmetry breaking such as the SK model [22].

Acknowledgements - The code for numerical integration was kindly given by K. Myazaki, whom we warmly thank for his technical support. We also thank G. Biroli and J.-P. Bouchaud for useful and stimulating discussions, as well as L. Berthier and J.-M. Luck for useful comments on the manuscript.

\section{REFERENCES}

[1] P. G. Debenedetti and F. H. Stillinger, Nature 410, 259 (2001). 
[2] A. J. Kovacs, Adv. Polym. Sci. 3, 394 (1963).

[3] K. Johnason et al., Phys. Rev. Lett. 81, 3243 (1998).

[4] M. M. Hurley and P. Harrowell, Phys. Rev. E 52, 1694 (1995) ; C. Bennemann et al., Nature 399, 246 (1999), C. Donati et al., J. Non-Cryst. Solids 307, 215 (2002) ; S. Whitelam, L. Berthier and J. P. Garrahan, Phys. Rev. Lett. 92, 185705 (2004) ; L. Berthier, Phys. Rev. E 69, 020201 (2004) ; G. Biroli and J.-P. Bouchaud, Europhys. Lett. 67, 21 (2004).

[5] W. Götze, in "Liquids, freezing and the glass transition", Proceedings of Les Houches summer school (1989).

[6] L. F. Cugliandolo, and J. Kurchan, Phys. Rev. Lett. 71, 173 (1993) ; L. F. Cugliandolo, and J. Kurchan, Progress. in Theor. Phys.126, 407 (1997).

[7] L. F. Cugliandolo, in "Slow relaxations in condensed matter", proceedings of Les Houches summer school (2002).

[8] J. Kurchan and L. Laloux, J. Phys. A 29, 1929 (1996).

[9] E. Vincent et al., in "Proceedings of the Stiges Conference on Glassy Systems", (1996), cond-mat/9607224

[10] M. Cloitre, R. Borrega and L. Leibler, Phys. Rev. Lett. 85, 4819 (2000) ; R. J. M. d'Arjuzon, W. Frith and J. Melrose, Phys. Rev. E 67, 061404 (2003).

[11] L. C. E. Struik, Physical Aging in Amorphous Polymers and Other Materials, Elsevier, Houston (1978) ; M. Ocio, M. Alba and J. Hammann, J. Physique Lett. (France) 46, L1101 (1985) ; M. Alba et al., J. Appl. Phys. 61, 3683 (1987).

[12] J.-P. Bouchaud et al., in "Recent progress in random magnets", A.P. Young ed., World Scientific (1997).

[13] M. P. Kennet and C. Chamon, Phys. Rev. Lett. 86, 1622 (2001).

[14] A. Crisanti and H.-J. Sommers, Z. Physik B 87, 341 (1992).

[15] L. F. Cugliandolo and P. Le Doussal, Phys. Rev. E 53, 1525 (1996).

[16] B. Kim and A. Latz, Europhys. Lett. 53, 660 (2001).

[17] W. Zippold, R. Kühn and H. Horner, Eur. Phys. J. B 13, 531 (2000).

[18] A. Andreanov and A. Lefèvre, unpublished.

[19] L. F. Cugliandolo and D. S. Dean, J. Phys. A 28, 4213 (1995).

[20] G. Biroli et al., cond-mat/0605733 (2006).

[21] G. Ben Arous, A. Dembo and A. Guionnet, cond-mat/0409273 (2004).

[22] D. Sherrington and S. Kirkpatrick, Phys. Rev. Lett. 35, 1792 (1975). 